\title{
GLOMERULONEFRITIS MESANGIOCAPILAR TIPO II Y DEGENERACIÓN MACULAR
}

\section{MESANGIOCAPILLARY GLOMERULONEPHRITIS TYPE II AND MACULAR DEGENERATION}

\author{
ASENSIO-SÁNCHEZ VM ${ }^{1}$, ASENSIO-SÁNCHEZ MJ ${ }^{2}$, RODRÍGUEZ-DELGADO B ${ }^{3}$, \\ GARCÍA-HERRERO E ${ }^{3}$, CABO-VAQUERA V ${ }^{3}$, GARCÍA-LOYGORRI C ${ }^{2}$
}

\begin{abstract}
RESUMEN
Caso clínico: Se describe una mujer de 34 años diagnosticada por biopsia de una glomerulonefritis mesangiocapilar tipo II (GMC II) y con un fondo de ojo similar al que se puede describir en un paciente con degeneración macular asociada a la edad (DMAE).

Discusión: Todos los pacientes con GMC II deben ser evaluados periódicamente por un oftalmólogo para controlar las complicaciones retinianas.

Palabras clave: Glomerulonefritis mesangiocapilar tipo II, degeneración macular, drusas, lipodistrofia, DMAE.
\end{abstract}

\begin{abstract}
Case report: We report a 34-year-old woman with biopsy proven type II mesangiocapillary glomerulonephritis (MCG II) who had an ophthalmic fundal appearance similar to that seen in patients with agerelated macular degeneration (ARMD).

Discussion: All patients with MCG II should be reviewed regularly by an ophthalmologist to assess and treat any retinal complications (Arch Soc Esp Oftalmol 2007; 82: 43-46).

Key words: Mesangiocapillary glomerulonephritis type II, macular degeneration, drusen, lipodystrophy, ARMD.

semejantes a las drusas retinianas (2,3). La mayoría de los pacientes con GMC tipo II desarrollan con la progresión de su enfermedad pérdida visual severa, a veces, irreversible. Sin embargo, la retinopatía y su asociación con GMC tipo II generalmente no es establecida por los especialistas que tratan a estos pacientes. Describimos el caso de una mujer joven con GMC tipo II que desarrolló en la evolución de su patología todos los componentes típicos de la enfermedad.
\end{abstract}

Recibido: 11/5/06. Aceptado: 18/1/07.

Hospital General. Medina del Campo. Valladolid. España.

1 Doctor en Medicina.

2 Doctor en Medicina. Servicio de Nefrología.

3 Licenciado en Medicina.

Correspondencia:

V.M. Asensio Sánchez

Hospital General Servicio Castellano-Leonés de Salud

Servicio de Oftalmología

Medina del Campo (Valladolid)

España

E-mail: vasensio@hmdc.sacyl.es 


\section{CASOS CLÍNICOS}

Mujer de 26 años remitida a la consulta de oftalmología en septiembre de 2005 desde el servicio de nefrología por disminución indolora bilateral de la visión mayor en el ojo izquierdo (OI) de varias semanas de evolución. En mayo 2001 fue diagnosticada con biopsia renal de glomerulonefritis mesangiocapilar tipo II (membranoproliferativa) asociada a lipodistrofia parcial. En la exploración física destacaba una mujer envejecida para su edad con aumento de los surcos nasogenianos por pérdida de grasa, aunque cuando fue explorada en la consulta, presentaba un edema generalizado por insuficiencia renal terminal (fig. 1). La agudeza visual máxima en el ojo derecho (OD) era de 0.5 y en el OI de 0.15 difícil. La biomicroscopía anterior no presentaba datos relevantes. El estudio retiniano mostró en la zona central depósitos de apariencia uniforme, semejantes a drusas duras que alrededor de la mácula eran de mayor tamaño y agrupables, con apariencia semisólida (fig. 2). En el angiograma se observó hiperfluorescencia de transmisión de la coroides en la zona de las drusas y en tiempos tardíos no hubo pérdida de contraste pero sí se evidenciaron las alteraciones del epitelio pigmentario macular (fig. $3)$. En noviembre de 2005 se le realizó un transplante renal falleciendo a los 15 días por un fallo multiorgánico.

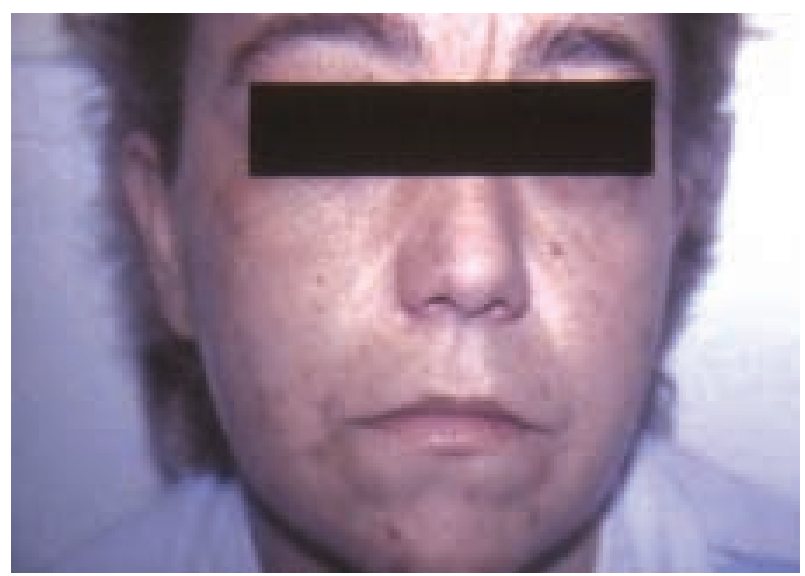

Fig. 1: Aspecto facial muy típico de los pacientes con glomerulonefritis mesangiocapilar tipo II. La lipodistrofia es por pérdida de grasa facial. El caso presentado tiene coloración de piel y edema de insuficiencia renal final.

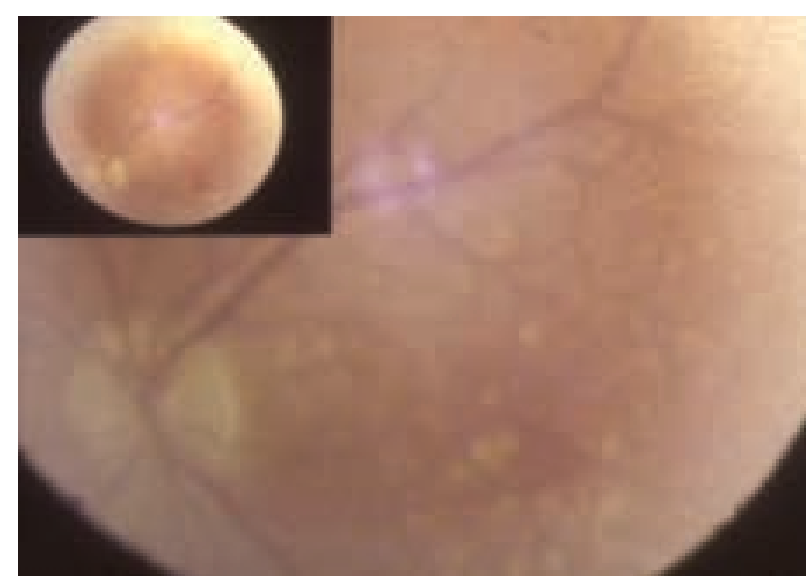

Fig. 2: Retinografía ojo izquierdo: ampliación del polo posterior en la paciente con glomerulonefritis mesangiocapilar tipo II indistinguible del fondo de ojo de una $D M A E$. En el inserto superior retinografía completa.

\section{DISCUSIÓN}

Las enfermedades glomerulares en su conjunto constituyen el origen de la insuficiencia renal terminal en un tercio de los pacientes tratados con diálisis o trasplante renal (1). La glomerulonefritis mesangiocapilar tipo II (membrano-proliferativa) forma parte de un grupo de procesos que presentan como común denominador un exceso de células (proliferación) en el glomérulo y el tipo al que nos referimos se caracteriza por un engrosamiento de la pared capilar secundario a un depósito denso lineal en el interior de la membrana basal formado fundamentalmente por C3 (1). La GMC II afecta principalmente a niños en edad escolar y adultos jóvenes

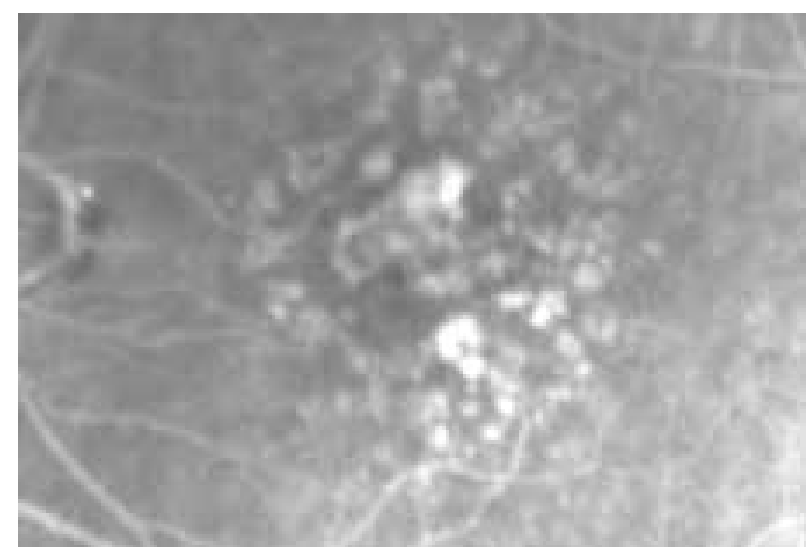

Fig. 3: Angiografía ojo izquierdo: acumulación de contraste en tiempos tardios como si existiera un desprendimiento del epitelio pigmentario. 
y es ligeramente más frecuente en mujeres que en hombres (1). La presentación clínica (que influye en su pronóstico) puede ser como proteinuria asintomática, hematuria recurrente, síndrome nefrótico o simulando una nefritis aguda. El pronóstico suele ser desfavorable y la mayoría mueren por uremia, hipertensión o por problemas secundarios al transplante dentro de los 10 años del comienzo (1-3). Los pacientes con GMC II generalmente tienen una lipodistrofia parcial y como característica de la enfermedad un descenso del C3 persistente (1-3). La mayoría de estos pacientes también tienen depósitos subretinianos clínicamente semejantes a drusas que son histopatológicamente idénticos a los depósitos de la membrana basal glomerular $(2,3)$. Suelen tener buena visión al inicio de la enfermedad pero progresivamente se produce una disminución de la visión nocturna y pérdida de la agudeza visual. Con la evolución la presencia de drusas es evidente y en un tercio de los pacientes se desarrolla neovascularización, especialmente en la región macular y con una evolución semejante a las membranas de la degeneración macular asociada a la edad (DMAE) $(2,3)$. La paciente que describimos presenta características clínicas y angiográficas indistinguibles de DMAE salvo la edad. PicKering MC et al (4) demostraron que la activación incontrolada de C3 origina GMC en el ratón deficiente en el factor $\mathrm{H}$. Hageman et al (5) publicaron que una variación en el gen del factor $\mathrm{H}(\mathrm{HF} 1 / \mathrm{CFH})$ incrementaba dramáticamente el desarrollo de DMAE y GMC tipo II. El factor $\mathrm{H}$ es un componente clave que regula la vía alternativa del complemento y todas estas investigaciones llevan a considerar al gen del factor $\mathrm{H}$ únicamente la descripción de tipo II y la DMAE. El objetivo de este trabajo no es una patología poco frecuente (GMC II) sino llamar la atención a oftalmólogos y nefrólogos que este tipo de pacientes precisan estudios oftalmológicos periódicos para evitar la pérdida de visión pudiendo beneficiarse de las nuevas terapias antiangiogénicas.

\section{BIBLIOGRAFÍA}

1. Sharpstone P, Trafford JA. Correlaciones anatomoclínicas. In: Sharpstone P, Trafford JA. Tópicos en enfermedades renales. Enfermedad renal glomerular. Madrid: Saned; 1983; 21-37.

2. Colville D, Guymer $R$, Sinclair RA, Savige J. Visual impairment caused by retinal abnormalities in mesangiocapillary (membranoproliferative) glomerulonephritis type II («dense deposit disease»). Am J Kidney Dis 2003; 42: E2-E5.

3. O'Brien C, Duvall-Young J, Brown M, Short C, Bone M. Electrophysiology of type II mesangiocapillary glomerulonephritis with associated fundus abnormalities. $\mathrm{Br} J$ Ophthalmol 1993; 77: 778-780.

4. Pickering MC, Cook HT, Warren J, Bygrave AE, Moss J, Walport MJ, et al. Uncontrolled C3 activation causes membranoproliferative glomerulonephritis in mice deficient in complement factor $H$. Nat Genet 2002; 31: 424428.

5. Hageman GS, Anderson DH, Johnson LV, Hancox LS, Taiber AJ, Hardisty LI, et al. A common haplotype in the complement regulatory gene factor $\mathrm{H}(\mathrm{HF} 1 / \mathrm{CFH})$ predisposes individuals to age-related macular degeneration. Proc Natl Acad Sci USA 2005; 102: 7227-7232. 